Behrend und Beer, Trioxydihydromethyluracil. 115

\title{
II. Ueber Trioxydihydromethyluracil;
} von Robert Behrend und Karl Beer.

Das Trioxydihydromethyluracil<smiles>CC1NCCCC1(O)C(=O)O</smiles>

ist zuerst von Behrend und Grünewald ') durch Umsetzung von Dibromoxymethyluracil mit Kali in wässeriger Lösung dargestellt. Später gelang es Behrend, den Körper in fester Form durch Einengen der Lösung im Vacuum $z u$ isoliren, und Beer hat ihn dann einer eingehenderen Lntersuchung unterworfen. Er fand zunächst, dass die Verbindung in $z$ wei verschiedenen Formen, $\alpha$ - and $\beta$-Trioxydihydromethyluracil, vorkommt, welche nicht nur verschiedene physikalische Eigenschaften haben, sondern sich auch beim Erhitzen verschieden verhalten. Behrend und Osten ${ }^{2}$ ) zeigten schliesslich, dass der Körper am bequemsten durch Oxydation von Aminomethyluracil mit Bromwasser bereitet werden kann und gaben eine genauere Beschreibung des Körpers. Insbesondere stellten sie die chemische Verschiedenheit der $\alpha$ - und $\beta$-Form durch Darstellung verschiedener Derivate sicher und ermittelten die Bedingungen, unter denen die beiden Formen in einander übergehen.

Zur Ergänzung sollen hier einige Angaben aus der Rostocker Inauguraldissertation von Beer nachgetragem werden.

1) Diese Annalen 323, 181.

2) Diese Anualen 343, 136. 
Analysen des $\alpha$-Trioxydihydromethyluracils:

I. $0,1710 \mathrm{~g}$ ergaben $0,1995 \mathrm{~g} \mathrm{CO}_{2}$ und $0,0830 \mathrm{~g} \mathrm{H}_{2} \mathrm{O}$.

II. $0,1700 \mathrm{~g} \quad, \quad 0,1995 \mathrm{~g} \mathrm{CO}_{2} \quad, 0,0842 \mathrm{~g} \mathrm{H}_{2} \mathrm{O}$.

III. $0,1389 \mathrm{~g} \quad, \quad 0,1600 \mathrm{~g} \mathrm{CO}_{2}, 0,0676 \mathrm{~g} \mathrm{H}_{2} \mathrm{O}$.

IV. $0,1785 \mathrm{~g}, 0,1878 \mathrm{~g} \mathrm{CO} \quad, 0,0845 \mathrm{~g} \mathrm{H}_{2} \mathrm{O}$.

IVa. $0,1740 \mathrm{~g}$ verloren bei $110^{\circ} \quad 0,0325 \mathrm{~g} \mathrm{H}_{2} \mathrm{O}$.

IVb. $0,1878 \mathrm{~g}$ ergaben $23,25 \mathrm{ccm}$ Stickgas bei $12^{\circ}$ and $754,5 \mathrm{~mm}$ Druck.

V. $0,2895 \mathrm{~g}$ ergaben $0,3240 \mathrm{~g} \mathrm{CO}_{2}$ und $0,1400 \mathrm{~g} \mathrm{H}_{2} \mathrm{O}$.

Berechnet für $\mathrm{C}_{5} \mathrm{H}_{8} \mathrm{~N}_{2} \mathrm{O}_{5}+\mathrm{H}_{2} \mathrm{O}$.

Gefunden:

$\begin{array}{lrcrrrr} & & \text { I } & \text { II. } & \text { III. } & \text { IV. } & \text { V. } \\ 2 \mathrm{H}_{2} \mathrm{O} & 18,56 & - & - & - & 18,68 & - \\ \mathrm{C} & 30,83 & 31,83 & 32,00 & 31,41 & 31,40 & 30,52 \\ \mathrm{H} & 5,15 & 5,39 & 5,50 & 5,41 & 5,26 & 5,38 \\ \mathrm{~N} & 14,43 & - & - & - & 14,59 & -\end{array}$

\section{Analysen des $\beta$-Trioxydihydromethyluracils.}

I. $0,4835 \mathrm{~g}$ verloren bei $105-100^{0} 0,0885 \mathrm{~g} \mathrm{H}_{2} \mathrm{O}$.

II. $0,1600 \mathrm{~g} \quad " \quad, 101^{0} 0,0299 \mathrm{~g} \mathrm{H}_{2} \mathrm{O}$.

$0,1770 \mathrm{~g}$ ergaben $0,2025 \mathrm{~g} \mathrm{CO}_{2}$ und $0,0790 \mathrm{~g} \mathrm{H}_{2} \mathrm{O}$.

$0,1500 \mathrm{~g}, \quad 18,80 \mathrm{ccm}$ Stickgas bei $17,50 \mathrm{u} .758 \mathrm{~mm}$ Druck.

Berechnet für $\mathrm{C}_{5} \mathrm{H}_{8} \mathrm{~N}_{2} \mathrm{O}_{5}+\mathrm{H}_{2} \mathrm{O}$.

Gefunden:

I. II.

$\begin{array}{lrcr}2 \mathrm{H}_{2} \mathrm{O} & 18,56 & 18,30 & 18,69 \\ \mathrm{C} & 30,93 & - & 31,20 \\ \mathrm{H} & 5,15 & - & 4,95 \\ \mathrm{~N} & 14,43 & - & 14,47\end{array}$

Die Phenylhydrazinverbindung des $\beta$-Trioxydihydromethyluracils wurde auf die von $O \operatorname{sten}^{3}$ ) für die Gewinnang der $\alpha$-Verbindung beschriebene Art bereitet.

Aus $1,9 \mathrm{~g} \beta$-Trioxydihydromethyluracil wurden $2,1 \mathrm{~g}$ Phenylhydrazinverbindung erhalten. Die schwach gelblich gefärbten Krystalle schmolzen nach dem Umkrystallisiren aus Alkohol bei $124-126^{\circ}$ unter Zersetzung. Bei Behandeln mit Alkali spalteten sie Phenylhydrazin ab, welches durch Ueberführung in das bei $152^{\circ}$ schmelzende Benzaldehydphenylhydrazon identifirt wurde. Es liegt also kein Phenylhydrazon sondern ein nach der Gleichung

8) a. a. 0 . 
Behrend und Beer, Trioxydihydromethyluracil. 117

$\mathrm{C}_{5} \mathrm{H}_{8} \mathrm{~N}_{2} \mathrm{O}_{5}+\mathrm{C}_{6} \mathrm{H}_{8} \mathrm{~N}_{2}=\mathrm{C}_{11} \mathrm{H}_{14} \mathrm{~N}_{4} \mathrm{O}_{4}+\mathrm{H}_{2} \mathrm{O}$ entstandenes Hydrazid vor.

$0,1250 \mathrm{~g}$ gaben $0,2235 \mathrm{CO}_{2}$ und $0,0625 \mathrm{H}_{2} \mathrm{O}$.

$0,1890 \mathrm{~g} \quad, \quad 34,2 \mathrm{ccm}$ feucht. Stickgas b. $19,5^{\circ}$ u. $748 \mathrm{~mm}$ Druck.

Bereehnet für $\mathrm{C}_{11} \mathrm{H}_{14} \mathrm{~N}_{4} \mathrm{O}_{4}$

$$
\begin{array}{lr}
\mathrm{C}=49,62 & 48,76 \\
\mathrm{H}=5,26 & 5,55 \\
\mathrm{~N}=21,06 & 20,50 .
\end{array}
$$

Acetoxalursaures Kali.

Das neutrale acetoxalursaure Kali wurde zuerst durch Oxydation der aus Dibromoxymethyluracil bereiteten Lösung von Trioxydihydromethyluracil bei Gegenwart von Kaliumbicarbonat erhalten.

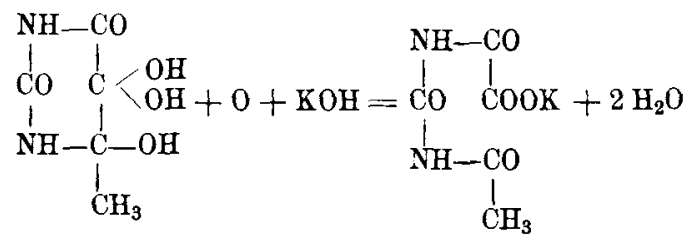

$6 \mathrm{~g}$ Dibromoxymethyluracil wurden unter Beobachtung der von Behrend und (trünewald ${ }^{4}$ ) angegebenen Vorsichtsmassregeln in Kalilauge gelöst und bei etwa $23^{\circ}$ mit einer Lösung von 2,1 $\mathrm{g}$ Kaliumpermanganat und $2.7 \mathrm{~g}$ Kaliumbicarbonat in $50-60 \mathrm{~g}$ Wasser oxydirt. Nach Beendigung der Oxydation (etwa 11/2 Stunden) wurde der Braunstein mittels eines Pukallfilters abgesaugt. Die Lösung (etwa $150 \mathrm{ccm}$ ) schied innerhalb 48 Stunden $0,7 \mathrm{~g}$ neutrales acetoxalursaures Kali in nadelförmigen Krystallen ab, welche sich bei $214-216^{\circ}$ unter Braunfärbung zersetzen.

A us dem auf $12 \mathrm{ccm}$ eingedampften Filtrate schieden sich Krystalle ab, denen heisser Alkohol 0,11 g Acetylharnstoff vom Schmelzp. 212-2140 entzog.

4) Diese Annalen 323, 196. 


\section{Behrend und Beer, Trioxydihylromethyluracil.}

Bei einem zweiten Versuche wurden $0,6 \mathrm{~g}$ neutrales acetoxalursaures Kali vom Schmelzp. $214-216^{\circ}$ gewonnen.

Bei einem dritten Versuche wurde das Filtrat vom Braunstein mit Salzsäure angesäuert. Bereits nach kurzem Stehen begann die Ausscheidung von saurem acetoxalursaurem Kali. Die nach 6 Stunden abgesaugten Krystalle wogen $1,07 \mathrm{~g}$, der Zersetzungspunkt lag bei $201-203^{0}$.

Das neutrale acetoxalursaure Kali krystallisirt mit zwei Molekeln Wasser, welche im Exsiccator neben Chlorcalcium nicht entweichen, wohl aber neben concentrirter Schwefelsäure oder beim Krhitzen auf $120^{\circ}$. An der Luft wird das Krystallwasser vollständig wieder aufgenommen, selbst im Chlorcalciumexsiccator nimmt das entwässerte Salz schnell an Gewicht zu.

I. $0,2230 \mathrm{~g}$ verloren bei $12000,0320 \mathrm{~g}$.

II. $0,1630 \mathrm{~g} \quad " \quad, 12000,0245 \mathrm{~g}$ u. gaben $0,0570 \mathrm{~K}_{2} \mathrm{SO}_{4}$.

III. $0,1945 \mathrm{~g}, "$ im Vacuum über Schwefelsäure $0,0282 \mathrm{~g}$ und gaben $0,0670 \mathrm{~K}_{2} \mathrm{SO}_{4}$.

Berechnet für $\mathrm{C}_{5} \mathrm{H}_{5} \mathrm{~N}_{2} \mathrm{O}_{5} \mathrm{~K}+2 \mathrm{H}_{2} \mathrm{O}$

Gefunden:

$\begin{array}{lccc} & \text { I. } & \text { II. } & \text { III. } \\ \mathrm{H}_{2} \mathrm{O}=14,52 & 14,35 & 15,03 & 14,50 \\ \mathrm{~K}=15,73 & - & 15,71 & 15,47\end{array}$

$0,1775 \mathrm{~g}$ wasserfreie Substanz gaben $0,1830 \mathrm{CO}_{2}$ und $0,0420 \mathrm{H}_{2} \mathrm{O}$. $0,1815 \mathrm{~g} \quad$ " "20,25 $\mathrm{cm}$ feuchtes Stickgas bei 120 und $762 \mathrm{~mm}$ Druck.

$\begin{array}{cc}\text { Berechnet für } \mathrm{C}_{5} \mathrm{H}_{5} \mathrm{~N}_{2} \mathrm{O}_{5} \mathrm{~K}, & \text { Gefunden } \\ \mathrm{C}=28.30 & 28,12 \\ \mathrm{H}=2,36 & 2,63 \\ \mathrm{~N}=13,21 & 13,29\end{array}$

Die mit Salzsäure angesänerte Lösung des Salzes scheidet nicht die freie Säure, sondern saures acetoxalursaures Kali ab. Aus 0,2 $\mathrm{g}$ wurden $0,1 \mathrm{~g}$ saures Salz vom Schmelzp. $201^{\circ}$ gewonnen.

Die Analyse des sauren acetoxalursauren Kali gab folgende Zahlen. Die im Vacuum über Schwefelsäure getrocknete Substanz verlor bei $150^{\circ}$ kein $1 \mathrm{~W}$ asser. 
Behrend und Beer, Trioxydihydromethyluracil. 119

$0,1847 \mathrm{~g}$ gaben $0,0417 \mathrm{~K}_{2} \mathrm{SO}_{4}$.

$0,1875 \mathrm{~g} \quad, \quad 0,2135 \mathrm{CO}_{2}$ und $0,0485 \mathrm{H}_{2} \mathrm{O}$.

$0,1365 \mathrm{~g} \quad, \quad 16,75 \mathrm{~cm}$ feuchtes Stickgas bei $12^{\circ}$ und $751 \mathrm{~mm}$ Druck.

$\begin{array}{cc}\text { Berechnet für } \mathrm{C}_{5} \mathrm{H}_{5} \mathrm{~N}_{2} \mathrm{O}_{5} \mathrm{~K}+\mathrm{C}_{5} \mathrm{H}_{6} \mathrm{~N}_{2} \mathrm{O}_{5} & \text { Gefundeu } \\ \mathrm{K}=10,13 & 10,14 \\ \mathrm{C}=31,09 & 31,03 \\ \mathrm{H}=2,85 & 2,87 \\ \mathrm{~N}=14,50 & 14,39\end{array}$

Suspendirt man das saure Salz in Wasser und setzt Kaliumbicarbonat hinzı, so löst es sich unter Kohlensäureentwicklung und gleich darauf scheidet sich das neutrale Salz ab. Aus $1 \mathrm{~g}$ saurem Salz wurden $0,5 \mathrm{~g}$ neutrales vom Schmelzp. $212-214^{\circ}$ gewonnen.

$0,1685 \mathrm{~g}$ entwässertes Sal\% gaben $0,0680 \mathrm{~g} \mathrm{~K}_{2} \mathrm{SO}_{4}$, entsprechend $18.12 \%$ Kalium, berechnet $18,45 \%$.

Dass das acetoxalursaure Kali beim Erhitzen mit Wasser oder Kali ausschliesslich Acetylharnstoff und Oxalsäure, nicht aber Oxalursäure und Essigsäure giebt, ist bereits von $O$ ffe $^{5}$ ) mitgetheilt.

Das acetoxalursaure Kali wurde auch durch Oxydation vom reinen ${ }^{-}$- und $\beta$-Trioxydihydromethyluracil bei Gegenwart von Kaliumbicarbonat dargestellt. Die Versuchsbedingungen hat 0 sten ${ }^{6}$ ), welcher die Versuche wiederholte, beschrieben.

$2 \mathrm{~g}$ a-Trioxydihydromethyluracil gaben $0,57 \mathrm{~g}$ saures acetoxalursaures Kali vom Schmelzp. 199_-2000.

$0,1520 \mathrm{~g} *$ gaben $0,0325 \mathrm{~K}_{2} \mathrm{SO}_{4}$ entsprechend $9,60 \%$ Kalium

$q_{2} 1550 \mathrm{~g}^{*} \quad, \quad 0,0345 \mathrm{~K}_{2} \mathrm{SO}_{4} \quad, \quad 9,99 \% \quad$,

Berechnet sind $10,13 \%$ Kalium.

$2 \mathrm{~g} \beta$-Trioxydihydromethyluracil gaben $0,6 \mathrm{~g}$ saures acetoxalursau res Kali vom Zersetzungspunkt 198-2000.

$0,1560 \mathrm{~g} *$ gaben $0,0335 \mathrm{~g} \mathrm{~K}_{2} \mathrm{SO}_{4}$ entsprechend $9,64 \%$ Kalium.

* Über Schwefelsäure im Yacuum getrocknet.

5) Diese Aunalen 353, 273.

b) a. a. 0 . 
120 Behrend und Beer, Trioxydihydromethyluracil.

\section{C-Acetallantursäure.}

Beim Erhitzen auf $105-110^{\circ}$ verliert das $\alpha$-Trioxydihydromethyluracil zwei Molekeln Wasser und geht in ein gelbes Pulver über, welches sich bei $140-145^{\circ}$ zersetzt, in Wasser und in verdünnten Säuren fast unlöslich ist, sich aber in concentrirter Salpeter- oder Schwefelsäure und in Alkalien leicht löst. Bei der Oxydation mit Kaliumpermanganat konnte weder acetoxalursaures noch oxalursaures Kali oder Acetylharnstoff aufgefunden werden. Durch Chromsäure wurde der Körper selbst in der Wärme kaum angegriffen.

$\beta$-Trioxydihydromethyluracil verliert bei $105-110^{\circ}$ ebenfalls 2 Molekeln Wasser, geht dabei aber in die syrupförmige Acetallantursäure über.

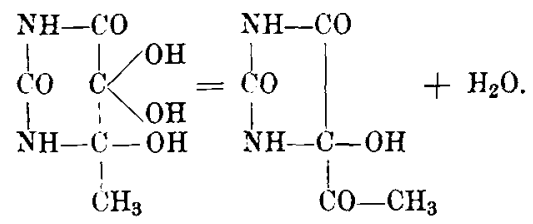

Die Säure konnte nicht in krystallinischem Zustande gewonnen werden, sie entsteht auch, wenn $\operatorname{man} \alpha$-oder $\beta$-Trioxydihydromethyluracil in neutraler oder schwach alkalischer wässeriger Lösung einige Zeit erwärmt und dann im Exsiccator zur Trockene bringt. .In Wasser löst sie sich leicht mit schwach saurer Reaction. Die Lösung färbt mit Ammoniak neutralisirte Eisenchloridlösung intensiv blan. Silbernitratlösung wird schon in der Kälte nach kurzer Zeit reducirt. Krystallisirbare Salze konnten nicht dargestellt werden, ebensowenig ein Ester durch Behandlung mit Alkali und Dimethylsulfat. Bei der Einwirkung von Kssigsäureanhydrid entstand ein dunkelbraunes Harz.

Dagegen gelang die Darstellung eines krystallinischen Phenylhydrazons. 
$0,8 \mathrm{~g} \beta$-Trioxydihydromethyluracil wurden bei $105^{\circ}$ zur Gewichtsconstanz gebracht, der entstandene Syrup in Wasser gelöst und mit $0,45 \mathrm{~g}$ Phenylhydrazin allmählich unter Umrühren vermischt. Das Hydrazon schied sich als gelbe Krystallmasse ab, welche abgesaugt, mit Alkohol ausgekocht und mit tether gewaschen wurde. Die gelblich gefärbten Krystalle bräunten sich bei $160^{\circ}$ und schmolzen unter Zersetzung bei $219-220^{\circ}$. Die Ausbeute betrug $0,62 \mathrm{~g}$. In Wasser, Alkohol, Aether und Benzol ist der Körper fast unlöslich, Kalilauge spaltet kein Phenylhydrazin ab.

$0,1334 \mathrm{~g}$ gaben $0,2610 \quad \mathrm{CO}_{2}$ und $0,0630 \quad \mathrm{H}_{2} \mathrm{O}$.

$0,1480 \mathrm{~g}, \quad 27,60 \mathrm{ccm}$ feucht. Stickgas bei 70 ॥. $767 \mathrm{~mm}$ Druck.

$\begin{array}{cr}\text { Berechnet für } & \text { Gefunden } \\ \mathrm{C}_{11} \mathrm{H}_{12} \mathrm{~N}_{4} \mathrm{O}_{3} & \\ \mathrm{C}=\mathbf{5 3 , 2 3} & 53,86 \\ \mathrm{H}=4,84 & 5,24 \\ \mathrm{~N}=\mathbf{2 2 , 5 8} & 22,73\end{array}$

Im Gegensatz zu den 'Trioxydihydromethyluracilen giebt die Acetallantursäure bei der Oxydation mit Kaliumpermanganat unter keinen Umständen Acetoxalursäure oder Acetylharnstoff sondern stets nur Oxalursäure und Fssigsäure.

$1,4 \mathrm{~g} \quad \beta$-Trioxydihydromethyluracil wurden durch Erhitzen auf $110^{\circ}$ in Acetallantursäure übergeführt, in $10 \mathrm{ccm}$ Wasser gelöst und unter Kühlung mit einer Lösung von $0,75 \mathrm{~g}$ Kaliumpermanganat (entsprechend 1 Atom Sauerstoff) und $0,95 \mathrm{~g}$ Kaliumbicarbonat in $30 \mathrm{ccm}$ Wasser versetzt. Im Filtrat vom Braunstein konnte Oxalsäure nicht nachgewiesen werden. Beim Kindampfen der neutralisirten Lösung auf $10 \mathrm{ccm}$ schieden sich $0,32 \mathrm{~g}$ Krystalle vom Zersetzungspunkt 235-2370 ab. Die Mutterlauge wurde im Vacuum über Schwefelsäure zur Trockene gebracht. Beim Auskochen beider Krystallisationen mit Alkohol ging kein Acetylharnstoft in Lösung, die zweite Krystallisation hinterliess beim Aufnehmen 
122 Behrend und Beer, Trioxydihydromethyluracil.

mit wenig Wasser noch 0,05 g hrystalle vom /ersetzungspunkt $234-235^{\circ}$.

Die schwerlöslichen Krystalle erwiesen sich als oxalursaures Kali. Sie waren frei von Oxalsäure, gaben aber beim Erwärmen mit Chlorcalcium und Ammoniak oxalsauren Kalk. Beim Versetzen mit Salzsäure schieden sich die schwerlöslichen Knollen von Oxalursämre ab.

$0,1205 \mathrm{~g}$ verloren bei $110^{\circ} 0,0110 \mathrm{~g}$ und gaben $0,0550 \quad \mathrm{~K}_{2} \mathrm{SO}_{4}$ entsprechend $9.13 \% \mathrm{H}_{2} \mathrm{O}$ und $20,507 \mathrm{Ka}-$ lium; berechnet für $\mathrm{C}_{3} \mathrm{H}_{3} \mathrm{~N}_{2} \mathrm{O}_{4} \mathrm{~K}+\mathrm{H}_{2} \mathrm{O} \quad 9.57 \% \mathrm{H}_{2} \mathrm{O}$ und $20,79 \%$ Kalium.

Bei einem zweiten Versuch wurde dieselbe Menge Acetallantursäure ebenso oxydirt, das Filtrat rom Braunstein im Vacuum über Schwefelsäure zur Trockene gebracht. Auch hier entzog heisser Alkohol dem Rückstande keinen Acetylharnstoff, dagegen wurden $0, \tilde{5} 0 \mathrm{~g}$ oxalsäurefreies oxalursaures Kali rom Zersetzungspunkt $236-238^{\circ}$ isolirt.

Bei der Oxydation der tcetallantursäure mit Chromsäure entsteht, wie auch $O$ sten bestätigte. Parabansäure.

$2 \mathrm{~g} \beta$-Trioxydihydromethyluracil wurden in teetallantursäure übergeführt und die Säure durch Zusatz einer Lösung von $0,8 \mathrm{~g}$ Chromsäure in $5 \mathrm{ccm} W$ asser in der Kälte oxydirt. Nach kurzem Stehen schieden sich aus der Lösung $0.6 \mathrm{~g}$ Parabansäure in Blättchen ab, die sich bei 235-237 zersetzten. Die Lauge und das Waschwasser gaben im Perforationsapparat von Partheil an Aether noch $0,16 \mathrm{~g}$ Parabansäure ab. Beide Krystallisationen wurden aus wenig Wasser umkrystallisirt und zeigten dann den Zersetzungspunkt $240^{\circ}$.

Mit Chlorcalcium und Ammoniak erwärmt, gab die Lösung einen Niederschlag von oxalsaurem Kalk.

$0,1285 \mathrm{~g}$ gaben $0.1510 \quad \mathrm{CO}_{2}$ und $0.0215 \quad \mathrm{H}_{2} \mathrm{O}$ entsprechend $32,05 \% \mathrm{C}$ und $1.86 \% \mathrm{H}$, während für $\mathrm{C}_{3} \mathrm{H}_{2} \mathrm{~N}_{2} \mathrm{O}_{3}$ $31,58 \% \mathrm{C}$ und $1,75 \% \mathrm{H}$ berechnet werden. 